\title{
The Role of Interleukin-1 Beta C-511T as a Modifier Polymorphism in Cryopyrin-associated Periodic Syndromes
}

\author{
(1) Berk Özyılmaz, (1) Taha Reşid Özdemir \\ University of Health Sciences, Tepecik Training and Research Hospital, Clinic of Genetic Diagnosis Center, Izmir, Turkey
}

\begin{abstract}
Aim: Cryopyrin-associated Periodic Syndromes (CAPS) are a subgroup of the Periodic fever syndromes, caused by mutations in the NLRP3 gene. NLRP3 gene mutations can cause three clinically different phenotypes. It is known that even the same mutations in the NLRP3 gene can cause different phenotypes. To investigate this situation, we have constructed a hypothesis that if an individual with the Interleukin-1 Beta

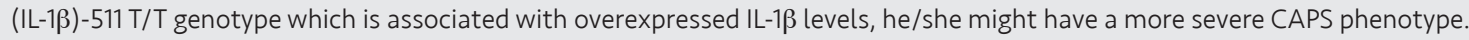

Materials and Methods: Thirty-six NLRP3 Exon three variant-positive patients with detailed clinical data and 30 healthy controls were selected for the IL-1 $\beta$ genotype investigation. For the analysis of IL-1 $\beta$-511 allele, the SNP rs1143634 was genotyped using the TaqMan 5'-exonuclease allelic discrimination assay.

Results: Neither the Muckle Wells Syndrome patients (severe phenotype) with a p.Val198Met mutation nor symptomatic patients with the p.Gln703Lys variant showed an increased IL-1 $\beta$-511T/T genotype frequency.

Conclusion: We suggest that IL-1 $\beta-511$ T/T polymorphism is not a modifying factor regarding the clinical severity of CAPS patients. However, to expand this theory and in order to find other modifying genetic factors, other polymorphisms of IL-1 $\beta$ or other genes in the inflammasome pathway such as caspase-1 or ASC should be analyzed.
\end{abstract}

Keywords: NLRP3, polymorphism, cryopyrin, IL-1 $\beta$, association

\section{Introduction}

Cryopyrin-Associated Periodic Syndromes (CAPS) are a subgroup of the periodic fever syndromes (PFSs) which are characterized by episodic fever and abdominal pain attacks (1). In addition to these, some severe neurological or systemic complications can result in life-threatening situations (2). CAPS are inherited in an autosomal dominant fashion with an estimated prevalence of $1 / 360.000$ people and they are caused by mutations in the NLRP3 gene (3).
The NLRP3 gene encodes cryopyrin (NALP3) protein. When activated by the NACHT domain, NALP3 recruits ASC and caspase- 1 and creates inflammasome. Inflammasome increases an inflammation by the activation and secretion of interleukin (IL)-18 and IL-1 beta (IL-1ß). The gain of function mutations in the NACHT domain of the NLRP3 protein leads to the overproduction of IL-1 $1 \beta$ by the activation of inflammasome and this causes fever, recurrent exaggerated systemic inflammatory response and the other systemic features of CAPS (4). 
Since most of the mutations in the NLRP3 gene are located in exon 3, which encodes the NACHT domain, the basic diagnostic strategy of CAPS includes sequence analysis of at least the exon 3 of the gene (5). NLRP3 gene mutations, even some identical mutations, can cause 3 clinically different phenotypes:

\section{Familial Cold Autoinflammatory Syndrome}

Familial Cold Autoinflammatory Syndrome (FCAS) is the mildest form of these 3 conditions. Cold exposure triggers a urticarial rash and fever. Conjunctivitis, arthralgia, fatigue, myalgia, and headaches are also common symptoms. Secondary amyloidosis is uncommon, unlike the other types of CAPS (6).

\section{Muckle-Wells Syndrome}

Muckle-Wells syndrome (MWS) is a more severe type of CAPS. Progressive sensorineural hearing loss and renal failure due to amyloidosis are serious complications of MWS (6).

\section{Chronic Infantile Neurological Cutaneous and Articular Disease or Neonatal Onset Multisystemic Inflammatory Disease}

Chronic infantile neurological cutaneous and articular/ neonatal onset multisystemic inflammatory disease (CINCA/NOMID) is the most severe form of CAPS. The urticarial rash starts just after birth. Apart from severe inflammatory symptoms, dysmorphic features, skeletal problems, central nervous system findings and eye involvement can also be seen (4).

As in many single gene disorders, identical mutations in NLRP3 can lead to different phenotypes (FCAS, MWS and CINCA/NOMID) in CAPS. It is known that single gene disorders caused by the same mutation in the same gene can be affected by background modifiers (7). Since the main pathogenic mechanism underlying CAPS is an overproduction of IL-1 $\beta$, and the IL-1 $\beta$ gene contains several single nucleotide polymorphisms, we hypothesized that if an individual with a specific IL-1 $\beta$ polymorphism is producing more IL-1 $\beta$ than others, he/she might have a more severe CAPS phenotype.

IL-1 $\beta$ polymorphisms have been investigated previously in other conditions and one of the polymorphisms, C-511T, which is located in the promoter region of the $I L-1 \beta$ gene, has been associated with increased IL-1 $\beta$ production in T/T state (8-10). Thus, we decided to investigate whether there is a role of IL-1 $\beta$ C-511T polymorphism in modifying disease severity.

\section{Materials and Methods}

The study was approved by the Local Research Ethics Committee University of Health Sciences, İzmir Tepecik Training and Research Hospital (approval number: 2018/14-12, date: 22.11.2018). Written informed consent was obtained from the patients or the parents of the patients and members of the healthy control group.

Thirty-six NLRP3 Exon 3 variant-positive patients whom we had previously reported in molecular genetic evaluation of periodic fever syndromes and 30 healthy controls were selected for the IL-1 $\beta$ genotype investigation.

To investigate whether there is a role of IL-1ß C-511T polymorphism in modifying disease severity, the patients with an NLRP3 variant were classified:

The patients with the p.Val198Met mutation (13 patients) were classified as either FCAS (7 patients) or MWS (6 patients).

The patients with the p.Gln703Lys variant (21 patients) were classified as either asymptomatic ( 8 patients) or symptomatic (13 patients).

For the investigation of the role of the IL-1 $\beta$ genotype, C-511T polymorphism was analyzed. For the analysis of IL-1 $\beta$-511 allele, the SNP rs1143634 was genotyped using the TaqMan 5'-exonuclease allelic discrimination assay. Allelespecific fluorescence was measured with a real-time PCR system.

\section{Results}

The heterozygous T/C genotype of IL-1 $1 \beta$ was the most common genotype both in patients with the NLRP3 variants and the healthy control groups. The homozygous $\mathrm{C} / \mathrm{C}$ was the second most common genotype in both groups.

When evaluated in terms of allele frequency, the $C$ allele was the most common allele both in patients with the NLRP3 variants and the healthy control group.

As for the analysis of those patients with the p.Val198Met mutation, genotype frequencies of IL-1 $1 \beta$ C-511T showed that $T / T$ allele was unexpectedly higher in those patients with FCAS (less severe phenotype) compared to those with MWS (severe phenotype) and the healthy control group ( $28.6 \%$ vs $0 \%$ and $20 \%$ ).

In those patients with the p.Gln703Lys variant, IL-1 $\beta$ 511, T/T allele was again unexpectedly higher in the asymptomatic patients compared to the symptomatic group $(12.5 \%$ vs $0 \%)$. 
The genotypes and allele frequencies of IL-1 $1 \beta$ C-511T in those patients with the NLRP3 variants and the healthy control samples are shown in Table I.

The comparison of genotypes according to clinical severity is summarized in Table II.

\section{Discussion}

The main pathogenic mechanism underlying CAPS is an overproduction of IL-1 $1 \beta$. The gain of function mutations in the NACHT domain of the NLRP3 protein leads to the overproduction of IL-1 $\beta$ by the activation of inflammasome and this causes fever, recurrent exaggerated systemic inflammatory response and the other systemic features of CAPS (6). In CAPS, although there is a clinical classification, a lack of correlation exists between genotype and phenotype.

It is well known that the same variants can lead to different phenotypes and this association can be explained by variable penetrance and expressivity. Researches have shown that variable phenotypes can be caused by a number of factors, such as modifier genes, environmental factors, allelic variations and complex genetic and environmental interactions (11). Modifier genes can affect transcription, change gene expressions and they are also capable of altering the effects of mutations (7).

In CAPS, the effects of background genetic factors such as other PFS-related genes (MEFV, TNFRSF1A and MVK) are possibly leading to these variable phenotypes. This suggestion was supported by several recent studies that have reported the coexistence of MEFV and NLRP3, TNFRSF1A or MVK gene variants that caused synergistic effects $(12,13)$. This selection of patients with mutations in multiple autoinflammatory genes show presentations which are challenging to delineate, such as atypical phenotypes (13).

One of the other possible explanations for variable and unexpected phenotypes in CAPS may be due to somatic mosaicism. Several studies have identified NLRP3 somatic mosaic mutations in children with CINCA/NOMID and MWS (14). Those patients with somatic mutations are probably missed by Sanger sequencing and will only be detected by the use of new technologies (14). Rowczenio et al. (15) suggested that when a mutation occurs in early embryonic life, it will cause mosaicism affecting more cells of the body. These data suggest that the emergence time and the level of mosaicism is associated with different phenotypes or different severity levels.

As a new candidate for these modifier genetic factors, we have constructed a hypothesis stating that since the main pathogenic mechanism is the overproduction of IL-1 $\beta$ and if an individual with a specific IL-1 $\beta$ polymorphism is producing more IL-1 $\beta$ than others, he/she might have a more severe CAPS phenotype. One of the IL-1 $1 \beta$ polymorphisms, C-511T, which is located in the promoter region of the IL-1 $\beta$ gene, has been associated with increased IL-1 $\beta$ production in T/T state $(8,10)$.

In our previously studied patient group, the same p.Val198Met mutation was causing FCAS (less severe) and MWS (more severe) phenotypes in different patients, and the same p.Gln703Lys variant was leading to asymptomatic and symptomatic patients (16). Thus, we have compared the IL-1 $13-511$ genotype within the p.Val198Met and the p.Gln703Lys groups and also compared them with the healthy controls. As a result, Neither the MWS patients (more severe phenotype) with p.Val198Met mutation nor

Table I. Interlekuin-1 beta-511 genotype and allele frequencies of patients and controls

\begin{tabular}{|c|c|c|c|c|c|c|}
\hline IL-1 $\beta$ & \multicolumn{5}{|c|}{ Patients with NLRP3 variants (36) } & Healthy controls (30) \\
\hline- & \multicolumn{5}{|c|}{ Genotype frequency } & Genotype frequency \\
\hline- & Total (36) & p.Q703K (21) & p.V198M (13) & p.R327Q (1) & p.K510N (1) & Total (30) \\
\hline $\mathrm{T} / \mathrm{T}$ & $3(8.3 \%)$ & 1 & 2 & 0 & 0 & $6(20 \%)$ \\
\hline $\mathrm{T} / \mathrm{C}$ & $17(47.3 \%)$ & 10 & 6 & 0 & 1 & $16(53.3 \%)$ \\
\hline $\mathrm{C} / \mathrm{C}$ & $16(44.4 \%)$ & 10 & 5 & 1 & 0 & $8(26.7 \%)$ \\
\hline- & - & - & - & - & - & - \\
\hline- & \multicolumn{5}{|c|}{ Allele frequency } & Allele Frequency \\
\hline- & Total (72) & - & - & - & - & Total (60) \\
\hline $\mathrm{T}$ & $23(31.9 \%)$ & - & - & - & - & $28(46.7 \%)$ \\
\hline C & $49(68.1 \%)$ & - & - & - & - & $32(53.3 \%)$ \\
\hline
\end{tabular}

IL-1 $\beta$ : Interlekuin-1 beta 
Table II. Interlekuin-1 beta-511 genotype and allele frequencies according to clinical severity

\begin{tabular}{|c|c|c|c|}
\hline \multirow[t]{2}{*}{ IL-1 $\beta$} & \multicolumn{2}{|l|}{ Patients with p.V198M Mutation (13 Patients) } & \multirow{2}{*}{$\begin{array}{l}\text { Healthy controls (30 Samples) } \\
-\end{array}$} \\
\hline & $\begin{array}{l}\text { Familial cold autoinflammatory syndrome } \\
\text { (7 Patients) }\end{array}$ & $\begin{array}{l}\text { Muckle-Wells Syndrome } \\
\text { (6 patients) }\end{array}$ & \\
\hline- & Genotype frequency & - & Total (30) \\
\hline$T / T$ & $2(28.6 \%)$ & $0(0 \%)$ & $6(20 \%)$ \\
\hline $\mathrm{T} / \mathrm{C}$ & $3(42.8 \%)$ & $3(50 \%)$ & $16(53.3 \%)$ \\
\hline $\mathrm{C} / \mathrm{C}$ & $2(28.6 \%)$ & $3(50 \%)$ & $8(26.7 \%)$ \\
\hline- & Allele frequency & - & Total (60) \\
\hline $\mathrm{T}$ & $7(50 \%)$ & $3(25 \%)$ & $28(46.7 \%)$ \\
\hline C & $7(50 \%)$ & $9(75 \%)$ & $32(53.3 \%)$ \\
\hline IL-1 $\beta$ & \multicolumn{2}{|l|}{ Patients with p.Q703K Variant (21 Patients) } & Healthy Controls (30 Samples) \\
\hline- & Asymptomatic (8 Patients) & Symptomatic (13 Patients) & - \\
\hline- & Genotype Frequency & - & Total (30) \\
\hline$T / T$ & $1(12.5 \%)$ & $0(0 \%)$ & $6(20 \%)$ \\
\hline $\mathrm{T} / \mathrm{C}$ & $3(37.5 \%)$ & $7(53.8 \%)$ & $16(53.3 \%)$ \\
\hline $\mathrm{C} / \mathrm{C}$ & $4(50 \%)$ & $6(46.2 \%)$ & $8(26.7 \%)$ \\
\hline- & Allele Frequency & - & Total (60) \\
\hline $\mathrm{T}$ & $5(31.3 \%)$ & $7(26.9 \%)$ & $28(46.7 \%)$ \\
\hline C & $11(68.7 \%)$ & $19(73.1 \%)$ & $32(53.3 \%)$ \\
\hline
\end{tabular}

IL-1 $\beta$ : Interlekuin-1 beta

symptomatic patients with the p.Gln703Lys variant showed an increased IL-1 $\beta-511 \mathrm{~T} / \mathrm{T}$ genotype frequency over the less severe phenotype groups and the healthy controls. Furthermore, IL-1 $\beta-511 \mathrm{~T} / \mathrm{T}$ genotype frequency in MWS patients with p.Val198Met mutation and symptomatic patients with the p.Gln703Lys variant was $0 \%$.

\section{Conclusion}

In this study, we couldn't confirm our hypothesis and we suggest that IL-1 $\beta-511 \mathrm{~T} / \mathrm{T}$ polymorphism is not a modifying factor regarding the clinical severity of CAPS patients. The failure of our hypothesis may be due to the small sample size and improper selection of the genetic modifier. We suggest that, to expand this theory and in order to find additional mechanisms, other modifying genetic factors such as other polymorphisms of IL-1 $\beta$ or other polymorphisms of the genes in the inflammasome pathway (caspase-1, ASC, etc.) should be analyzed.

Additionally, to obtain a full clinical interpretation, it is necessary to consider the possibility of somatic mosaicism and polygenic inheritance in molecular genetic evaluation by using next generation techniques.

\section{Ethics}

Ethics Committee Approval: The study was approved by the Local Research Ethics Committee University of Health Sciences, İzmir Tepecik Training and Research Hospital (approval number:2018/14-12, date: 22.11.2018).

Informed Consent: Written informed consent was obtained from the patients or the parents of the patients and members of the healthy control group.

Peer-review: Externally and internally peer-reviewed.

\section{Authorship Contributions}

Surgical and Medical Practices: B.Ö., T.R.Ö., Concept: B.Ö., T.R.Ö., Design: B.Ö., T.R.Ö., Data Collection or Processing: B.Ö., T.R.Ö., Analysis or Interpretation: B.Ö., T.R.Ö., Literature Search: B.Ö., T.R.Ö., Writing: B.Ö., T.R.Ö.

Conflict of Interest: The authors have no conflicts of interest relevant to this article to disclose.

Financial Disclosure: The authors have no financial relationships relevant to this article to disclose.

\section{References}

1. Marcuzzi A, Piscianz E, Kleiner G, et al. Clinical genetic testing of periodic fever syndromes. Biomed Res Int 2013;2013:501305. 
2. Smith DD. Periodic fever syndromes www.dermnetnz.org: Available from: http://www.dermnetnz.org/topics/periodicfever-syndromes/

3. Ling E, Ling G, Pinsk V. A case of cryopyrin-associated periodic fever syndrome due to Q703K mutation in the NLRP3 gene. Int Rheum Dis 2017;20:2233-5.

4. Giat E, Lidar M. Cryopyrin-associated periodic syndrome. Isr Med Assoc / 2014;16:659-61.

5. Shinar $Y$, Obici L, Aksentijevich I, et al. Guidelines for the genetic diagnosis of hereditary recurrent fevers. Ann Rheum Dis 2012;71:1599-605.

6. Levy R, Gérard L, Kuemmerle-Deschner J, et al. Phenotypic and genotypic characteristics of cryopyrin-associated periodic syndrome: A series of 136 patients from the Eurofever Registry. Ann Rheum Dis 2015;74:2043-9.

7. Kammenga JE. The background puzzle: How identical mutations in the same gene lead to different disease symptoms. FEBS I 2017;284:3362-73.

8. Chen $H$, Wilkins LM, Aziz $N$, et al. Single nucleotide polymorphisms in the human interleukin-1 $\beta$ gene affect transcription according to haplotype context. Hum Mol Genet 2006; 15:519-29.

9. Goldbach-Mansky R, Kastner DL. Autoinflammation: The prominent role of IL-1 in monogenic autoinflammatory diseases and implications for common illnesses. I Allergy Clin Immunol 2009;124:1141-9.
10. Hall SK, Perregaux DG, Gabel CA, et al. Correlation of polymorphic variation in the promoter region of the interleukin$1 \beta$ gene with secretion of interleukin-1 $\beta$ protein. Arthritis Rheum 2004;50:1976-83.

11. Lobo I. Same genetic mutation, different genetic disease phenotype. Nature Education, 2008:1.

12. Kubota $\mathrm{K}$, Ohnishi $\mathrm{H}$, Teramoto $\mathrm{T}$, et al. In vitro analysis of the functional effects of an NLRP3 G809S variant with the co-existence of MEFV haplotype variants in atypical autoinflammatory syndrome. I Clin Immunol 2013;33:325-34.

13. Timerman D, Frank NY. Novel double heterozygous mutations in MEFV and NLRP3 genes in a patient with familial Mediterranean fever. J Clin Rheumatol 2013;19:452-3.

14. Nakagawa K, Gonzalez-Roca E, Souto A, et al. Somatic NLRP3 mosaicism in Muckle-wells syndrome. A genetic mechanism shared by different phenotypes of cryopyrin-associated periodic syndromes. Ann Rheum Dis 2015;74:603-10.

15. Rowczenio DM, Gomes SM, Aróstegui II, et al. Late-onset cryopyrin-associated Periodic syndromes caused by somatic NLRP3 Mosaicism-UK single center experience. Front Immunol 2017;8:1410.

16. Ozyilmaz B, Kirbiyik $\mathrm{O}$, Koc A, et. Al. Molecular genetic evaluation of NLRP3, MVK and TNFRSF1A associated periodic fever syndromes. Int J Immunogenet. 2019 Aug;46:232-40. 